\title{
Transcriptional response to hypoxia of normal and rheumatoid arthritis synovial fibroblasts
}

\author{
M J Del Rey ${ }^{1 *}$, E Izquierdo ${ }^{1}$, A Usategui ${ }^{1}$, E Gonzalo ${ }^{1}$, F J Blanco ${ }^{2}$, F Acquadro ${ }^{3}$, J L Pablos ${ }^{1}$ \\ From 5th European Workshop on Immune-Mediated Inflammatory Diseases \\ Sitges-Barcelona, Spain. 1-3 December 2010
}

\section{Introduction}

Rheumatoid arthritis (RA) synovium is characterized by very low oxygen concentrations but it is not clear how this factor contributes to the disease process. Hypoxia has profound and variable effects on gene expression in different cell types but these effects have not been systematically analysed in human fibroblasts.

\section{Aim}

Characterize the changes on gene expression induced by hypoxia in healthy (HSF) and rheumatoid arthritis synovial fibroblasts (RASF).

\section{Materials and methods}

We used microarray Agilent G4112F expression profiling in total RNAs extracted from paired normoxic and hypoxic cultures from $6 \mathrm{HSF}$ and 6 RASF lines. P-value from paired Student's t-test with multiple testing Benjamini and Hochberg correction, and two-fold change in gene expression (up-regulation or down-regulation) were used to define a differentially regulated gene. Validation of microarray data was performed by quantitative RT-PCR. Finally, biological pathways enriched by hypoxia in RASF or HSF were identified using unsupervised Ingenuity Pathway Analysis.

\section{Results}

The number of genes differentially regulated by hypoxia was significantly higher in HSF compared to RASF, although the magnitude of the response (fold change) was similar for commonly up-regulated individual genes. A group of 278 transcripts was differentially and similarly regulated by hypoxia in RASF and HSF, whereas 107 and 309 transcripts were specifically regulated in
RASF and HSF respectively. In hypoxia, the number of transcripts differentially expressed by RASF compared to HSF was significantly higher (336) than in normoxia (223). Only 108 of the differentially expressed transcripts in RASF in normoxia remained different in hypoxia, whereas a larger group of 228 transcripts were only found differentially expressed in hypoxia. The main functional categories of genes significantly regulated by hypoxia were related with energy metabolism, including glucose transport, glycolisis and AMPK pathways. Multiple gene categories related with intracellular signal transduction, angiogenesis, immune and inflammatory pathways were also significantly modified.

\section{Conclusion}

These data demonstrate that hypoxia induces significant changes on gene expression in HSF and RASF, and identify differences between RASF and HSF profiles. The hypoxia-induced gene expression program in SF identifies new factors and pathways relevant to understand their contribution to the pathogenesis of chronic arthritis.

\section{Author details}

${ }^{1}$ Servicio de Reumatología, Instituto de Investigación Hospital 12 de Octubre, Madrid, Spain. ${ }^{2}$ Laboratorio de Investigación Osteoarticular y del Envejecimiento, INIBIC, Complejo Hospitalario Universitario A Coruña, A Coruña, Spain. ${ }^{3}$ Molecular Cytogenetics Group, Centro Nacional de Investigaciones Oncológicas, Madrid, Spain.

Published: 25 November 2010

doi:10.1186/1479-5876-8-S1-P31

Cite this article as: Del Rey et al:: Transcriptional response to hypoxia of normal and rheumatoid arthritis synovial fibroblasts. Journal of Translational Medicine 2010 8(Suppl 1):P31. 